\title{
Celiomesenteric trunk demonstrated by multi- -detector computed tomography angiography: two cases of a rare vascular variation
}

\author{
Ch. Rountas ${ }^{1}$, M. Fanariotis ${ }^{1}$, M. Vlychou ${ }^{1}$, D.L. Arvanitis ${ }^{2}$, I. Fezoulidis ${ }^{1}$, K. Vassiou ${ }^{1}, 2$ \\ ${ }^{1}$ Department of Anatomy, Faculty of Medicine, School of Health Science, University of Thessaly, Larissa, Greece \\ ${ }^{2}$ Department of Radiology, Faculty of Medicine, School of Health Science, University of Thessaly, Larissa, Greece
}

[Received: 24 January 2013; Accepted 7 February 2013]

\begin{abstract}
We present two cases of patients with celiomesenteric trunk in whom the celiac trunk and the superior mesenteric artery arise off a common vessel from the ventral part of the aorta, which was demonstrated by multi-detector (16 slices) computed tomography angiography (MDCTA) and confirmed by digital subtraction angiography (DSA). This is a very rare congenital vascular anomaly and its imaging demonstration is of great importance in several interventional procedures. These cases demonstrate the capability of MDCTA in the evaluation of abdominal aorta and its branches and shows that this method might replace diagnostic DSA. (Folia Morphol 2013; 72, 2: 171-175)
\end{abstract}

Key words: celiomesenteric trunk, multidetector computed tomography angiography, mesenteric arterial variation

\section{INTRODUCTION}

The celiac trunk usually arises from the ventral part of the aorta at the level of the $12^{\text {th }}$ thoracic vertebra and it branches off the left gastric, common hepatic and splenic arteries [10]. The superior mesenteric artery arises $1 \mathrm{~cm}$ below, at the level of the first lumbar vertebrae [1].

The celiomesenteric trunk (CMT) is a rare congenital vascular variation in which the celiac trunk and the superior mesenteric artery (SMA) arise off a common vessel from the ventral part of the aorta $[2,10,16]$. Its incidence is less than $1 \%$ of all celiomesenteric anomalies [4] and less than $2 \%$ of all individuals [16].

The anatomical knowledge and the imaging demonstration of the celiac trunk and its branches, and of the SMA is of great importance in cases of hepato-biliary pancreatic surgery [14] and interventional radiological treatments [14].

Digital subtraction angiography (DSA) has been the modality of choice in the evaluation of abdomi- nal aorta and its branches. Multi-detector computed tomography angiography (MDCTA) is a relatively new, fast and easy to perform imaging modality which can accurately demonstrate the abdominal aorta and its branches by replacing the use of DSA [4].

We describe two cases of common CMT demonstrated by MDCTA and confirmed by DSA.

\section{CASE REPORT 1}

A 45 year-old man was admitted to our hospital for transarterial chemoembolisation (TACE) because of hepatocellular carcinoma. Abdominal MDCTA was performed to detect the exact hepatic arterial anatomy before the treatment. The examination was performed on a 16-slice scanner (LightSpeed 16, General Electric, Milwaukee, WI, USA) with $1.25 \mathrm{~mm}$ collimation, $0.5 \mathrm{~s}$ rotation speed and 1.375 pitch. An automatic power injector was used to administer intravenously a $100 \mathrm{~mL}$ bolus of $300 \mathrm{mg} / \mathrm{mL}$ non-ionic iodinated contrast material at a rate of $4 \mathrm{~mL} / \mathrm{s}$. The 
scan delay was determined by using automated triggering hardware and dedicated software (SmartPrep; GE Healthcare). The scan was acquired during the arterial phase. The threshold enhancement trigger was set at $100 \mathrm{HU}$ in the abdominal aorta. Post processing volume rendering was applied for better appreciation of the vessels of the upper abdomen.

MDCTA revealed the presence of a common trunk divided to the celiac trunk and SMA (Fig. 1). The hepatic, splenic and left gastric arteries were depicted with normal origin form the celiac trunk and normal course. This was a short CMT with length less than $1 \mathrm{~cm}(0.8 \mathrm{~cm})$ although it was long enough not to be misdiagnosed as a common origin of the SMA and the celiac trunk.

DSA took place in terms of TACE (V 3000 Integris, Philips, Eindhoven, the Netherlands). The examination protocol included an aortogram of the abdominal aorta using a $5 \mathrm{~F}$ pigtail catheter (Cordis, Johnson \& Johnson, Miami, Florida, USA) with a transfemoral approach. Non-ionic contrast agent was injected into the aorta at a rate of $12 \mathrm{~mL} / \mathrm{s}$ (max. dose $25 \mathrm{~mL}$ ). Selective catheterisation of the celiac trunk was performed with a hydrophilic catheter (Cobra 1, Terumo, Belgium) in the posteroanterior and lateral view. The anatomical variant of the CMT was confirmed (Fig. 2).

\section{CASE REPORT 2}

An 80 year-old woman with a history of atrial fibrillation was admitted to the emergency department of our hospital because of abdominal pain, weakness and malaise. White blood cells count was markedly elevated $(30.000 / \mu \mathrm{L})$ along with increased D-dimer levels ( $2.1 \mathrm{mg} / \mathrm{L})$. An abdominal MDCTA was performed in order to exclude the possibility of SMA thrombosis. The examination was performed on a 16-slice scanner (LightSpeed 16, General Electric, Milwaukee, WI, USA) with $1.25 \mathrm{~mm}$ collimation, $0.5 \mathrm{~s}$ rotation speed and 1.375 pitch. An automatic power injector was used to administer intravenously $100 \mathrm{~mL}$ of $300 \mathrm{mg} / \mathrm{mL}$ non-ionic iodinated contrast material at a rate of $4 \mathrm{~mL} / \mathrm{s}$. The scan delay was determined by using automated triggering hardware and dedicated software (SmartPrep; GE Healthcare). The scan was acquired during the arterial phase. The threshold enhancement trigger was set at $100 \mathrm{HU}$ in the abdominal aorta.

Although no sign of SMA thrombosis was found, the MDCTA revealed a common CMT. The CMT after running a path of $7 \mathrm{~mm}$ was dichotomised into the SMA and the celiac trunk. The hepatic, splenic and
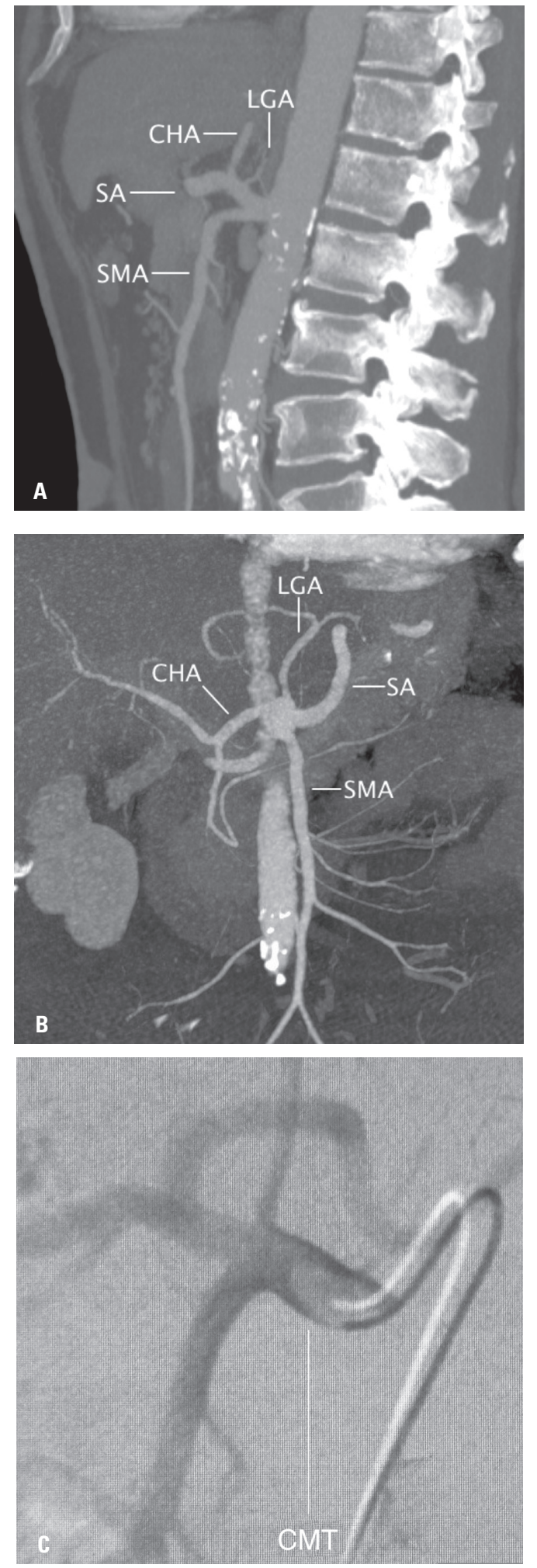

Figure 1. A 45 year-old man with hepatocellular carcinoma. Multiplanar image reformatting $(\mathbf{A})$ shows the common origin of the celiac trunk and superior mesenteric artery (SMA) from the ventral part of the aorta. Thick-slab maximum intensity projection (B) coronal computed tomography image demonstrates the left gastric artery (LGA), common hepatic artery (CHA), splenic artery (SA) and SMA arising from a short celiomesenteric trunk (CMT). Digital subtraction angiography (C) shows the CMT trunk. 

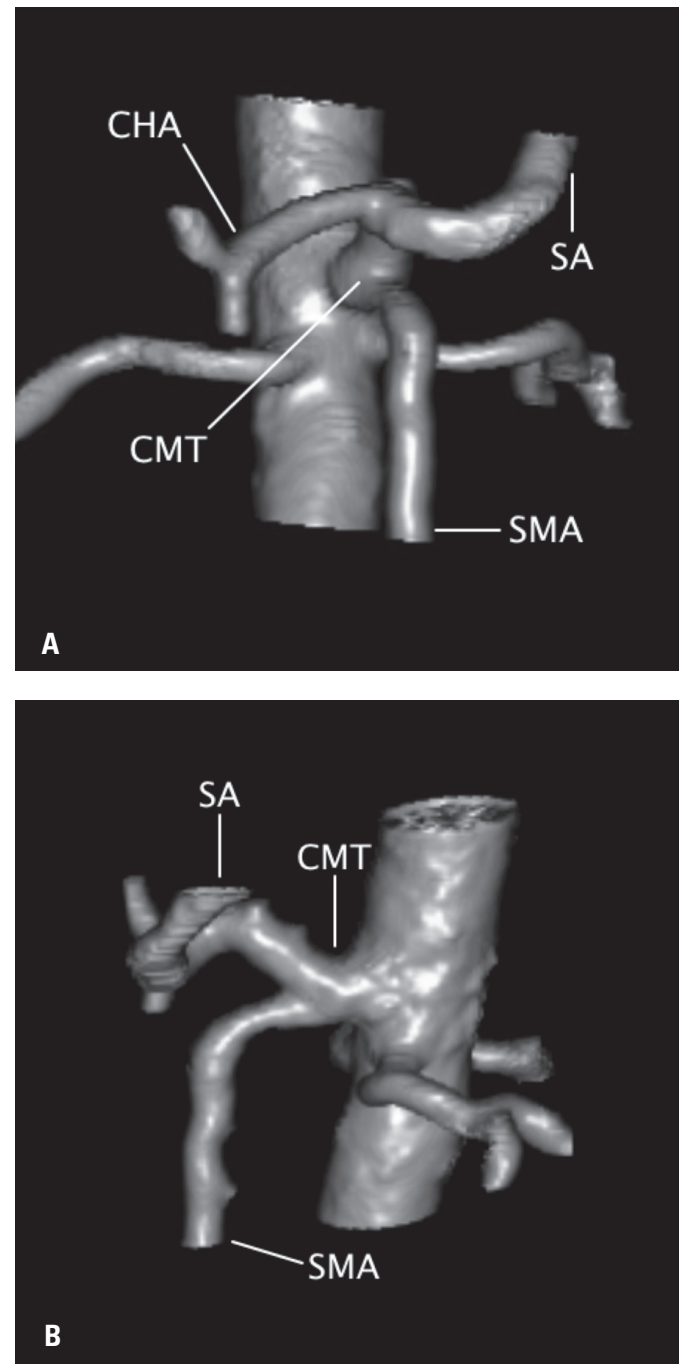

Figure 2. A, B. A 45 year-old man with hepatocellular carcinoma. $3 \mathrm{D}$ volume rendered images shows the superior mesenteric artery (SMA), the common hepatic artery (CHA) and the splenic artery (SA) arising from the celiomesenteric trunk (CMT).

left gastric arteries were depicted with normal origin form the celiac trunk and normal course. There was no misdiagnosing between CMT and common origin of the SMA and the celiac trunk. No subsequent DSA was performed to this patient.

The diameters of the aforementioned vessels are presented in Table 1 for both cases.

\section{DISCUSSION}

The celiac trunk and SMA provide a dual vascular supply to the majority of the abdominal viscera. Many anatomical variations of the celiac trunk have been described. In rare cases celiac trunk and SMA arise from the abdominal aorta as a common trunk, an anomaly called CMT, and it accounts for $1.5 \%$ of
Table 1. Diameters of abdominal arteries based on measurements that were performed on celiomesenteric trunk (CTA)

\begin{tabular}{lcc}
\hline \multirow{2}{*}{ Name of the artery } & \multicolumn{2}{c}{ Diameter [mm] } \\
\cline { 2 - 3 } & Case 1 & Case 2 \\
\hline CTA & 11.5 & 13.7 \\
Celiac trunk & 7 & 5.3 \\
Superior mesenteric artery & 6.2 & 6.2 \\
Common hepatic artery & 5.6 & 3.9 \\
Left gastric artery & 4.8 & 2 \\
Splenic artery & 8.6 & 3.2 \\
\hline
\end{tabular}

all celiomesenteric anomalies according to a review by $\mathrm{Yi}$ et al. [23].

CMT is usually asymptomatic and may be discovered incidentally during vascular surgery $[1,15]$, radiologic imaging [8], or cadaver's dissection [10]. Symptoms are rare and vary between unspecific abdominal pain and real abdominal angina, when associated to atherosclerosis [1]. Aneurysms and thrombosis of the CMT have also been reported $[6,13]$.

CMT is the result of developmental changes in the ventral arteries of the visceral organs. In embryonic development, abdominal visceral arteries develop from the primitive dorsal abdominal aorta through four roots: the left gastric artery, the hepatic artery, the splenic artery, and the SMA [12]. These roots are joined together by a ventral longitudinal anastomosis, called Lang's anastomose. Normally, a cleft appears on the anastomosis between the third and fourth root and the separation of the celiac trunk with the hepatic artery, the left gastric artery and splenic artery form the SMA is achieved [21]. Consequently, the origins of the celiac trunk and SMA from the abdominal aorta are separated. The persistence of the ventral longitudinal anastomosis accounts for the CMT [12].

Regarding comparative anatomy, Polguj et al. [17] described the presence of CMT in insectivora as the first visceral branch of the abdominal aorta. Also, CMT was found in other mammals, including guinea pig and sheep [5, 22]. The presence of this vessel in dogs was exceedingly rare $[5,22]$.

CMT may be further divided into 4 categories according to the arrangement of the arteries [9]. These categories are classified by Higashi and Hirai [7] as follows: Type I - a hepatosplenogastric trunk with trifurcation, Type II - the common hepatic artery arises from the CMT after the splenogastric trunk, Type III - the left gastric artery arises from the CMT before the hepatosplenic trunk, Type IV - separate 
origins of the gastric, splenic and hepatic arteries on the CMT. Our cases are classified as Type IV.

The length of CMT may vary from less than $1 \mathrm{~cm}$ to a few centimeters. In both cases presented in this article CMT's length was less than $1 \mathrm{~cm}$. In the literature there are 2 reports of the short CMT variant, the one detected on MDCTA [16] and the other discovered during anatomic dissection of a cadaver [3]. This variant may be underdiagnosed with angiography as it quite difficult to discern a short CMT from a common origin of the SMA and the celiac trunk, as the tip of the catheter may easily transverse the common trunk and enter the orifice of one of its branches [16]. This could explain the few reports of this variant to the literature [16].

The identification of this variation is of clinical significance because in the presence of CMT the abdominal viscera lack the collateral protection of the dual vascular supply of celiac trunk and SMA. The whole blood supply to the major abdominal viscera may be at risk in any disorder involving the CMT trunk, especially in cases of atherosclerosis or vascular interventional procedures complications [16] Furthermore, in patients with arterial variants there is an increased risk of hepatic artery complications after liver transplantation [2], as well as there has been reported increased complication rates during chemoembolisation in patients with variant of the mesenteric arterial anatomy [16].

In our cases the diagnosis of CMT was performed by MDCTA. To the best of the authors knowledge, only 3 other cases of CMT detected by MDCTA has been described $[11,16,23]$. DSA has been the gold standard for the evaluation of the abdominal aorta and its branches, for years. However, DSA is an invasive and uncomfortable procedure, with radiation exposure and possible complications like hemorrhage, thrombosis and dissection [19]. MDCTA is increasingly used in the evaluation of the abdominal arterial vascular system and because of its non-invasive nature and diagnostic accuracy it serves as a reliable alternative to DSA. Improvements in CT technology permit the acquisition of volumetric data of the entire abdomen in a single breath-hold with high-resolution isotropic voxels, and a 3-dimensional reconstruction of the abdomen is possible [18]. MDCT with multiplanar image reformatting delineates the course of small caliber vessels, facilitating the depiction of vascular anatomic variations [16]. In our clinical practice,

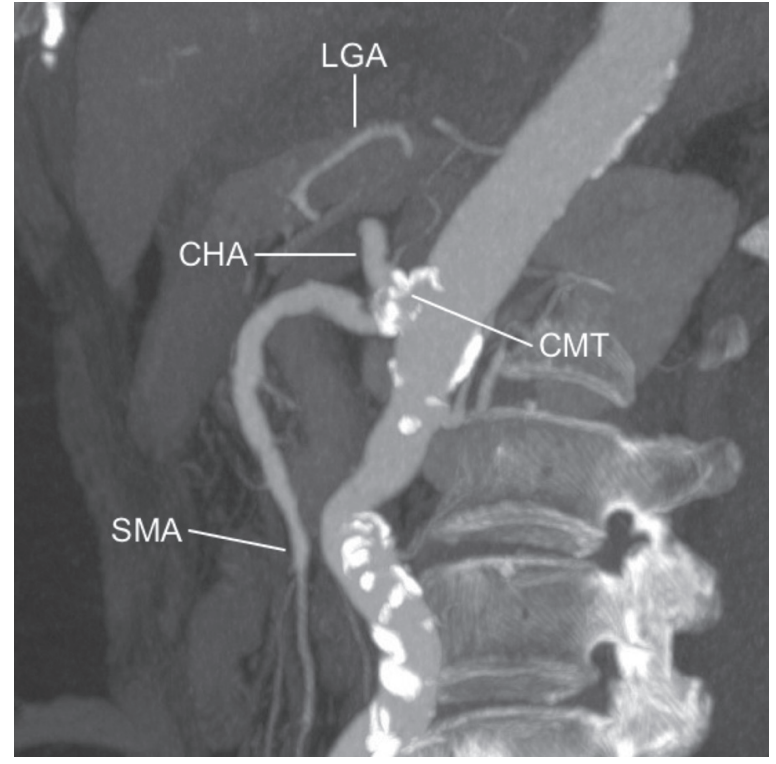

Figure 3. A 80 year-old woman with a celiomesenteric trunk (CMT). Thick-slab maximum intensity projection sagittal image shows common hepatic artery (CHA), left gastric artery (LGA) and superior mesenteric artery (SMA) originating from a short CMT.

vessels up to $1.5 \mathrm{~mm}$ in diameter can be identified with 16-slice MDCT, $1.25 \mathrm{~mm}$ collimation. Maximum intensity projection (MIP) was used in order to have a detailed overview of vascular anatomy (Fig. 3). There should be noted that axial source images should always be reviewed and compared with MIP reconstructions, as these are the basis of the diagnostic process [20]. Several anatomic vascular variations are described by means of MDCTA, confirmed by DSA, with preoperative clinical significance. The number of MDCTA studies is gradually increasing and, therefore, knowledge of normal and variant anatomic features has become very important.

\section{CONCLUSIONS}

In conclusion, the knowledge of this rare anomaly and its imaging demonstration is of importance in the presurgical evaluation of patients and MDCTA is an accurate, alternative to DSA, imaging modality for visualisation of CMT.

\section{REFERENCES}

1. Ailawadi G, Cowles RA, Stanley JC, Eliason JL, Williams DM, Colletti LM, Henke PK, Upchurch GR, Jr. (2004) Common celiacomesenteric trunk: aneurysmal and occlusive disease. J Vasc Surg, 40: 1040-1043.

2. Borley N (2005) Posterior abdominal wall and retroperitoneum. Gray's anatomy. 39th Ed. Churchill Livingstone, Edinburgh. 
3. Cavdar S, Sehirli U, Pekin B (1997) Celiacomesenteric trunk. Clin Anat, 10: 231-234.

4. De Cecco CN, Ferrari R, Rengo M, Paolantonio P, Vecchietti F, Laghi A (2009) Anatomic variations of the hepatic arteries in 250 patients studied with 64-row CT angiography. Eur Radiol, 19: 2765-2770.

5. Egund N, Olin T (1976) Catheterization and angiography of the abdominal arteries in the guinea-pig. Acta Radiol Diagn (Stockh), 17: 493-510.

6. Guntani A, Yamaoka T, Kyuragi R, Honma K, Iwasa K, Matsumoto T, Nishizaki T, Maehara Y (2011) Successful treatment of a visceral artery aneurysm with a celiacomesenteric trunk: report of a case. Surg Today, 41: 115-119.

7. Higashi N, Hirai K (1995) A case of the three branches of the celiac trunk arising directly from the abdominal aorta. Kaibogaku Zasshi, 70: 349-352.

8. Kara E, Celebi B, Yildiz A, Ozturk N, Uzmansel D (2011) An unusual case of a tortuous abdominal aorta with a common celiacomesenteric trunk: demonstrated by angiography. Clinics, 66: 169-171.

9. Katagiri H, Ichimura K, Sakai T (2007) A case of celiacomesenteric trunk with some other arterial anomalies in a Japanese woman. Anat Sci Int, 82: 53-58.

10. Lagoutte N, Facy O, Guiu B, Favier C, Cheynel N (2011) Celiacomesenteric trunk: a variation that must be known before aortic surgery. Clinics Practice, 1: e69. doi:10.4081/ /cp.2011.e69.

11. Lawler LP, Fishman EK (2001) Celiomesenteric anomaly demonstration by multidetector $\mathrm{CT}$ and volume rendering. J Comput Assist Tomogr, 25: 802-804.

12. Lin J (2005) Celiomesenteric trunk demonstrated by 3-dimensional contrast-enhanced magnetic resonance angiography. Hepatobiliary Pancreat Dis Int, 4: 472-474.
13. Lovisetto $F$, Finocchiaro De Lorenzi G, Stancampiano $P$, Corradini C, De Cesare F, Geraci O, Manzi M, Arceci F (2012) Thrombosis of celiacomesenteric trunk: report of a case. World J Gastroenterol, 18: 3917-3920.

14. Marcos A, Fisher RA, Ham JM, Shiffman ML, Sanyal AJ Luketic VA, Sterling RK, Posner MP (1999) Right lobe living donor liver transplantation. Transplantation, 68: 798-803.

15. Matsumoto K, Tanaka K, Ohsumi K, Nakamaru M, Obara H, Hayashi S, Kitajima M (1999) Celiomesenteric anomaly with concurrent aneurysm. J Vasc Surg, 29: 711-714.

16. Petscavage JM, Maldjian P (2007) Celiomesenteric trunk two variants of a rare anomaly. Australas Radiol, 51 (suppl. B): 306-309.

17. Polguj M, Gabryniak T, Topol M (2010) The right accessory hepatic artery; a case report and review of the literature. Surg Radiol Anat, 32:175-179.

18. Saba L, Mallarini G (2011) Anatomic variations of arterial liver vascularization: an analysis by using MDCTA. Surg Radiol Anat, 33: 559-568.

19. Sakamoto I, Hayashi K, Matsunaga N, Matsuoka Y, Uetani M, Fukuda T, Fujisawa H (1994) Aortic dissection caused by angiographic procedures. Radiology, 191: 467-471.

20. Smith CL, Horton KM, Fishman EK (2006) Mesenteric CT angiography: a discussion of techniques and selected applications. Tech Vasc Interv Radiol, 9: 150-155.

21. Tandler J (1904) Uber die Varietaten der Arteria coeliaca und deren Entwickelung. Anat Hefte, 25: 473-500.

22. Wustinger J (1978) Developmental anomaly of the hepatic artery in a sheep. Folia Morphol, 37: 99-102.

23. Yi SQ, Terayama H, Naito M, Hayashi S, Moriyama H, Tsuchida A, Itoh M (2007) A common celiacomesenteric trunk, and a brief review of the literature. Ann Anat, 189: 482-488. 